\title{
Perfil funcional de pacientes com Esclerose Lateral Amiotrófica ao longo de 14 meses de tratamento fisioterapêutico
}

\author{
Gabriele Natane de Medeiros Cirne, Luana Augusta Pimenta Bezerra, \\ Roberta de Oliveira Cacho, Thaiana Barbosa Ferreira, Fabrícia Azevêdo da Costa Cavalcanti \\ Departamento de Fisioterapia, Universidade Federal do Rio Grande do Norte - UFRN, Natal, RN, Brasil.
}

\begin{abstract}
Resumo: Introdução: A Esclerose Lateral Amiotrófica (ELA) é uma doença degenerativa e fatal, que provoca diretamente alterações na funcionalidade dos indivíduos acometidos. A fisioterapia assume um papel importante na reabilitação dessa doença, utilizando instrumentos de avaliação que acompanham a performance funcional. Objetivo: Investigar as alterações funcionais decorrentes da ELA por 14 meses em indivíduos com e sem tratamento fisioterapêutico. Método: Estudo de coorte realizado entre 2010 e 2013, na cidade de Natal-RN. Foram incluídos no estudo pacientes em diversos estágios da ELA, com ausência de patologias associadas e que estivessem realizando acompanhamento no Centro de Referência em Doença do Neurônio Motor/ELA desde 2010. As características funcionais foram categorizadas a partir dos itens motores da Medida de Independência Funcional (MIF), da Escala de Severidade de Fadiga (FSS) e da Escala de Avaliação Funcional da ELA (ALSFRS). Os participantes foram divididos em dois grupos: os que tinham atendimento fisioterapêutico (AF) e os que não tinham atendimento (GC), tendo sido avaliados longitudinalmente em três momentos distintos (primeiro dia de avaliação, quatro meses após o primeiro dia e dez meses após a segunda avaliação). Foi realizado o teste não paramétrico de Friedman, considerando-se significância $\mathrm{p}<0,05$. Resultados: Foi verificado significativo declínio funcional no $\mathrm{GC}(\mathrm{p}<0,05)$ mensurado pela ALSFRS. O GC também apresentou declínio funcional maior que o grupo AF a partir dos escores da MIF, no entanto sem relevância estatística. Não houve diferença para os escores da FSS. Conclusão: A fisioterapia pode ser um grande coadjuvante no retardo de declínio funcional em pacientes com ELA. Ressalta-se também a importância de escalas específicas para ELA, considerando-se o caráter progressivo e heterogêneo da doença.
\end{abstract}

Palavras-chave: Esclerose Amiotrófica Lateral, Reabilitação, Fisioterapia.

\section{Functional profile of ALS patients over 14 months of physical therapy}

\begin{abstract}
Introduction: Amyotrophic Lateral Sclerosis (ALS) is a degenerative and fatal disease that changes the individuals' functionality. Physical therapy plays an important role in ALS by using assessment tools to evaluate functional performance. Objective: To investigate functional changes caused by ALS through 14 months in individuals with and without physical therapy. Method: A cohort study conducted between 2010 and 2013 in the city of Natal-RN. The study included patients in various stages of ALS, with no associated pathologies and those who were being monitored by the Reference Center since 2010. Functional characteristics were categorized by applying the motor items of Functional Independence Measure (FIM), Fatigue Severity Scale (FSS) and ALS-Functional Rating Scale (ALSFRS). Participants were divided into two groups, those who performed physical therapy (FA) and those who didn't (CG). They were longwise evaluated in 3 different moments (1st day of assessment, four months after the first assessment and 10 months after the second evaluation). Nonparametric Friedman test was performed, considering $p<0.05$. Results: It was observed a significant functional decline in the control group $(p<0.05)$ measured by the
\end{abstract}

Autor para correspondência: Thaiana Barbosa Ferreira, Departamento de Fisioterapia, Universidade Federal do Rio Grande do Norte, CP 1524, Campus Universitário Lagoa Nova, Natal, RN, Brasil, e-mail: thata_fisio2007@yahoo.com.br

Recebido em Jun. 19, 2015; $1^{\text {a }}$ Revisão em Out. 16, 2015; $2^{\text {a }}$ Revisão em Jan. 26, 2016; $3^{\text {a }}$ Revisão em Mar. 15, 2016; Aceito em Mar. 17, 2016 
ALSFRS. The CG also presented functional decline greater than the AF group from FIM scores, however without statistical significance. There was no difference in the scores of FSS. Conclusion: Physical therapy can be a great adjunct in functional decline retardation in patients with ALS. It also emphasizes the importance of specific scales for ALS, considering the disease progressive and heterogeneous nature.

Keywords: Amyotrophic Lateral Sclerosis, Rehabilitation, Physical Therapy.

\section{Introdução}

A Esclerose Lateral Amiotrófica (ELA) é uma doença progressiva, degenerativa e fatal, que envolve os neurônios motores inferiores e superiores (MITCHEK; BORASIO, 2007; BANDEIRA et al., 2010) localizados no córtex primário, tronco cerebral e corno anterior da medula espinhal (WIJESEKERA; LEIGH, 2009).

As manifestaçôes clínicas iniciais da doença podem envolver sintomas bulbares, que incluem fraqueza da musculatura facial, perda de movimentos palatais, fasciculação de língua e lábios, problemas respiratórios, disfagia e disartria, ou sintomas apendiculares, que envolvem fraqueza de membros, movimentos lentificados, fasciculação e atrofia muscular. Entretanto, com a progressão da doença, o acometimento atingirá ambas as regiōes (MITCHEK; BORASIO, 2007; YORKSTON et al., 2004; COMIM et al., 2009).

Para alcançar a independência funcional, o indivíduo necessita da conexão de fatores cognitivos, comportamentais e sensório-motores, tendo por finalidade que as habilidades motoras sejam produzidas de forma efetiva (CESÁRIO; PENASSO; OLIVEIRA, 2006). Contudo, as doenças do neurônio motor afetam diretamente o controle muscular, provocando alteração na função e no bem-estar daqueles com esses diagnósticos (JENKINSON et al., 1999). A compreensão de fatores clínicos e epidemiológicos associados ao prejuízo funcional é de fundamental importância para a adoção precoce de medidas que possibilitem uma maior sobrevida e melhor qualidade de vida (COSMO; LUCENA; SENA, 2012).

Em muitos países, os indivíduos acometidos pela ELA são acompanhados em centros que se baseiam nos cuidados interdisciplinares, já que não existe cura para essa doença. Com isso, as pesquisas vêm demonstrando que os indivíduos com ELA acompanhados por equipe interdisciplinar em centros de referência apresentam melhor prognóstico (VAN DEN BERG, 2005).

Nesse sentindo, a fisioterapia assume um papel importante na área de reabilitaçâo dessa doença, podendo utilizar instrumentos de avaliaçáo funcional que classificam e acompanham a performance funcional
(KIMURA et al., 2006; KAUFMANN et al., 2005). Entretanto, ainda há poucos estudos que documentem a utilização de escalas de avaliação para a ELA; por isso, este trabalho investigou as alteraçôes físicas e funcionais decorrentes da ELA por 14 meses em indivíduos com tratamento fisioterapêutico e sem tratamento.

\section{Método}

Trata-se de um estudo de coorte realizado no período de 2010 a 2013. O estudo foi realizado no Centro de Referência em Doença do Neurônio Motor/ELA, do Hospital Universitário Onofre Lopes (HUOL), na cidade de Natal, Rio Grande do Norte, Brasil. Este centro de referência recebe pacientes com ELA de todas as regióes do Rio Grande do Norte e estados vizinhos. O Centro de Referência em Doença do Neurônio Motor/ELA é formado por uma equipe multidisciplinar composta por médico neurologista, nutricionistas, terapeuta ocupacional, psicólogo, fonoaudiólogo e fisioterapeuta para assistência respiratória e motora. Os pacientes eram acompanhados por esta equipe de forma que cada um dos profissionais realizavam avaliaçóes periódicas e forneciam orientaçôes individualizadas, que variavam de acordo com o quadro clínico do paciente. O papel da equipe de fisioterapia motora era principalmente acompanhar a evolução clínica e funcional, realizar orientaçóes para prevenir contraturas e deformidades, prescrever quanto a dispositivos para auxílio à mobilidade e orientar sobre a importância de o paciente buscar e realizar intervenção fisioterapêutica constante.

Foram incluídos, neste estudo, pacientes nos diversos estágios da ELA, com ausência de patologias associadas que pudessem acarretar sequelas cognitivas e funcionais além das provocadas pela ELA, tais como amputação e doenças crônicas severas (AVC, Parkinson, Alzheimer, etc.), e que estivessem realizando acompanhamento no referido centro desde 2010. Foram excluídos pacientes altamente incapacitados, que não puderam responder aos protocolos utilizados na pesquisa, ou aqueles que se recusaram a participar do estudo. 
Os participantes foram divididos em dois grupos: o grupo atendimento fisioterapêutico (AF) foi formado por pacientes que realizavam tratamento de fisioterapia motora em algum serviço de saúde do estado do Rio Grande do Norte com frequência mínima de uma vez por semana e que contemplasse abordagem de fisioterapia neurofuncional, com ênfase na reabilitação do aparelho locomotor. Já os pacientes que também eram acompanhados pelo Centro de Referência, porém que não realizavam tratamento de fisioterapia motora, formaram o grupo controle (GC). Dessa forma, este estudo náo abordou um protocolo específico de tratamento fisioterapêutico, mas buscou compreender as manifestaçóes físicas e funcionais destes pacientes a partir da submissão ou não a este tipo de tratamento especificamente. Os participantes foram avaliados longitudinalmente em três momentos distintos (primeiro dia de avaliação, quatro meses após o primeiro dia e dez meses após a segunda avaliação) e as avaliaçóes foram aplicadas em sessóes únicas, por dois pesquisadores previamente treinados.

Todos os pacientes envolvidos na pesquisa apresentavam diagnóstico clínico de ELA baseado nos critérios de diagnóstico "El Escorial", revisados por Brooks et al. (2000).

Os dados gerais dos participantes foram colhidos através de uma Ficha de Avaliação Neurológica, que envolveu dados pessoais do participante (nome completo, idade, sexo, endereço, telefone e estado civil) e dados clínicos (história da doença atual e se tinha atendimento fisioterapêutico).

A funcionalidade foi avaliada a partir da Medida de Independência Funcional (MIF), que avalia quantitativamente a carga de cuidados demandada por uma pessoa para a realização de atividades da vida diária, sendo estas motoras e cognitivas. A pontuação para cada atividade varia de 1 (dependência total) a 7 (independência completa); assim, o total varia de 18 a 126 (KEITH et al., 1987). Porém, neste estudo, foram utilizados, exclusivamente, os itens motores da MIF: mobilidade, locomoção, controle de esfíncteres e cuidados pessoais, dispensando assim a parte cognitiva deste protocolo, limitando-se então o escore mínimo igual a 13 e o máximo de 91 pontos.

Em seguida, foi aplicada a Escala de Severidade de Fadiga (FSS), instrumento que tem sido amplamente utilizado para avaliar a fadiga em diversas patologias, inclusive de caráter neurológico (PEREIRA; DUARTE, 2010). Composta por um questionário com nove itens, o paciente escolhe um número de 1 a 7 que melhor represente sua circunstância. A pontuação varia de 9 a 63, e a pontuaçáo igual ou acima que 28 pontos indica presença de fadiga (KRUPP et al., 1989; MILLER et al., 1999).

Por fim, foi aplicada a escala de avaliação funcional da ELA, a Amyotrophic Lateral Sclerosis Functional Rating Scale-ALSFRS. O questionário é composto por dez itens, classificados em uma escala de 0-4 pelo paciente e/ou profissional, na qual a máxima pontuação atingida é 40 pontos. Na ALSFRS, quanto maior a pontuaçáo, melhor a funcionalidade do paciente, avaliando a capacidade na realização de atividades, como: alimentação, autocuidado, deambulação e comunicação (CEDARBAUM et al., 1999; CEDARBAUM; STAMBLER, 1997; MIANO et al., 2004; OHASHI et al., 2001; STAMBLER, 1996).

Foi realizado o teste não paramétrico de Friedman, pois os dados não apresentaram distribuição normal, conforme o teste Lilliefors. Estas análises foram realizadas através do programa BioStatic 5.3, sendo consideradas significativas quando $\mathrm{p}<0,05$.

O estudo foi aprovado pelo Comitê de Ética em Pesquisa da Universidade Federal do Rio Grande do Norte (Parecer n. ${ }^{\circ}$ 167/2010) e todos os pacientes ou responsáveis assinaram o Termo de Consentimento Livre e Esclarecido, autorizando a participação no estudo.

\section{Resultados}

Foram avaliados dez pacientes com ELA, com média de idade de 54,7 $\pm 11,2$ anos, variando de 34 a 70 anos. Destes, um estudou até o Ensino Fundamental I (10\%), quatro estudaram até o Ensino Fundamental II (40\%), quatro até o Ensino Médio (40\%) e um, o Ensino Superior (10\%).

A caracterização dos indivíduos dos dois grupos (GC e AF) está apresentada na Tabela 1.

$\mathrm{Na}$ Tabela 2, são apresentadas as médias dos valores de cada escala nas três avaliaçóes e o seu

Tabela 1. Caracterização dos grupos da pesquisa.

\begin{tabular}{lcc}
\hline & GC & AF \\
\hline Participantes (n) & 6 & 4 \\
Sexo masculino (\%) & $83,3 \%$ & $25 \%$ \\
Idade (m \pm dp) & $58( \pm 8,8)$ & $49,75( \pm 13,9)$ \\
Tempo de & $5( \pm 2,8)$ & $6( \pm 6,16)$ \\
diagnóstico (m \pm dp) & & \\
\hline
\end{tabular}

n (número); m (média); dp (desvio padrão). 
Tabela 2. Variância das escalas: Medida de Independência Funcional (MIF), Escala de Severidade de Fadiga (FSS) e Amyotrophic Lateral Sclerosis Functional Rating Scale (ALSFRS).

\begin{tabular}{|c|c|c|c|c|c|c|c|c|}
\hline & \multicolumn{2}{|c|}{$\begin{array}{l}\text { 1. }{ }^{\mathrm{a}} \text { AVAL. } \\
(\mathrm{m} \pm \mathrm{dp})\end{array}$} & \multicolumn{2}{|c|}{$\begin{array}{l}\text { 2. }{ }^{a} \text { AVAL. } \\
(\mathrm{m} \pm \mathrm{dp})\end{array}$} & \multicolumn{2}{|c|}{$\begin{array}{l}\text { 3. }{ }^{\mathrm{a}} \text { AVAL. } \\
(\mathrm{m} \pm \mathrm{dp})\end{array}$} & \multicolumn{2}{|c|}{$\begin{array}{l}\text { Teste de Friedman } \\
\text { (p valor) }\end{array}$} \\
\hline & GC & AF & GC & AF & GC & AF & GC & AF \\
\hline MIF & $\begin{array}{l}70 \\
\pm 17,2\end{array}$ & $\begin{array}{l}75,2 \\
\pm 8,7\end{array}$ & $\begin{array}{l}64,1 \\
\pm 16,8\end{array}$ & $\begin{array}{c}77,2 \\
\pm 12,8\end{array}$ & $\begin{array}{c}55,8 \\
\pm 17,7\end{array}$ & $\begin{array}{c}76,2 \\
\pm 16,2\end{array}$ & 3 & 0,5 \\
\hline FSS & $\begin{array}{r}35,5 \\
\pm 13\end{array}$ & $\begin{array}{l}37,5 \\
\pm 20,4\end{array}$ & $\begin{array}{l}25,8 \\
\pm 15,6\end{array}$ & $\begin{array}{l}11 \\
\pm 4\end{array}$ & $\begin{array}{c}30,5 \\
\pm 7,2\end{array}$ & $\begin{array}{c}29 \\
\pm 16,1\end{array}$ & 1,75 & 2 \\
\hline ALSFRS & $\begin{array}{c}28,8 \\
\pm 9,1 \\
\end{array}$ & $\begin{array}{l}30 \\
\pm 8 \\
\end{array}$ & $\begin{array}{l}26,8 \\
\pm 9,3 \\
\end{array}$ & $\begin{array}{r}31,7 \\
\pm 4,5 \\
\end{array}$ & $\begin{array}{l}22,8 \\
\pm 8 \\
\end{array}$ & $\begin{array}{c}29,2 \\
\pm 8,5\end{array}$ & $6,33^{*}$ & 0,875 \\
\hline
\end{tabular}

$* \mathrm{p}<0,05$.

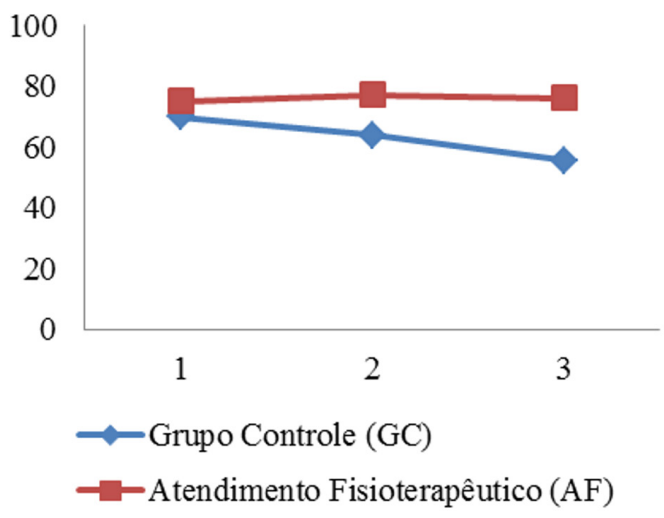

Figura 1. Variância nos valores da Medida de Independência Funcional (MIF).

desvio padrão em cada grupo. Não houve significância estatística nas escalas, exceto para a ALSFRS no GC.

$\mathrm{Na}$ Figura 1, observa-se que houve um declínio progressivo nos valores da MIF do GC, nas três avaliaçôes. Já no grupo AF, foi observada uma tendência à linearidade no escore da MIF, uma vez que, embora tenha havido um leve aumento na pontuação da primeira para segunda avaliação, o resultado da terceira avaliação diminuiu, mantendo valores próximos à primeira avaliação. A Figura 2 demonstra que o GC e o AF apresentaram-se inicialmente com valores próximos, seguidos de um declínio da primeira para a segunda avaliação, sendo mais notório no AF. Ao observar o curso da FSS da segunda para a terceira avaliação, o GC e o AF retornaram a valores próximos.

Já na Figura 3, é possível observar um declínio progressivo no escore da ALSFRS do GC e, embora tenha sido verificado também um discreto declínio da mesma escala do grupo AF, é observada novamente uma tendência à linearidade nos escores deste grupo, sendo esta diferença estatisticamente significativa.

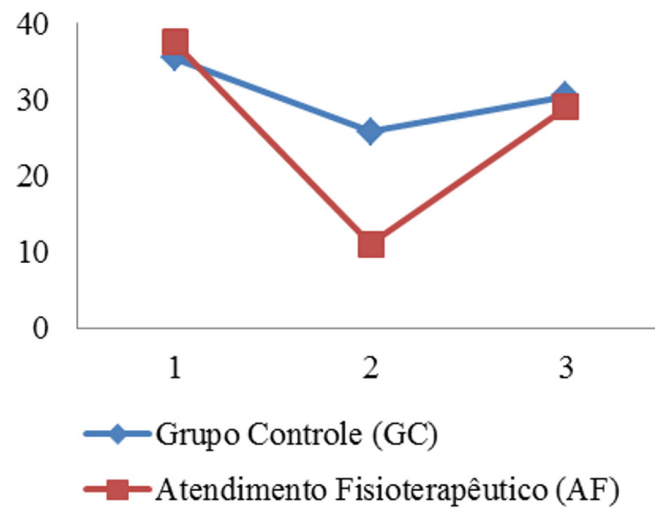

Figura 2. Variância nos valores da Escala de Severidade da Fadiga (FSS).

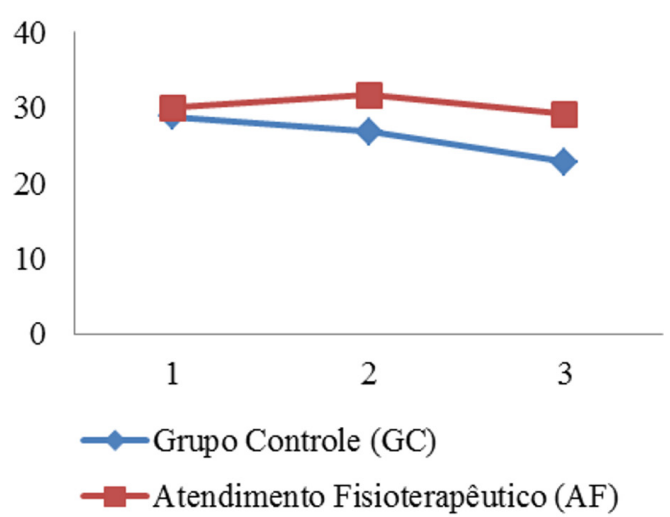

Figura 3. Variância nos valores da Amyotrophic Lateral Sclerosis Functional Rating Scale (ALSFRS).

\section{Discussão}

De acordo com os achados na literatura, há uma maior prevalência de ELA nos indivíduos do sexo masculino; em seu estudo, Pozza et al. (2006) afirmam que há uma proporção de 3:2. Estes dados condizem com os encontrados no presente estudo, em que houve um maior predomínio do sexo 
masculino. A idade variou de 34 a 70 anos e teve uma média de 54,7 anos. Tal resultado se assemelha também com o estudo de Bandeira et al. (2010), em que se avaliou a qualidade de vida de indivíduos acometidos pela ELA no período de janeiro de 2008 a dezembro de 2008; dos 16 indivíduos analisados, 13 eram homens, e a idade variou entre 39 e 80 anos. Outro estudo que corrobora com esta pesquisa é de Anequini et al. (2006), em que 60\% dos indivíduos avaliados eram do sexo masculino. Em contrapartida, Cosmo, Lucena e Sena (2012), em estudo transversal, observaram maior prevalência de ELA nos indivíduos do sexo feminino.

Para Shumway-Cook e Woollacott (2003), estratégias terapêuticas devem ser utilizadas para o processo de reabilitação motora em indivíduos com patologias neurológicas, uma vez que terá eficácia na aprendizagem e/ou reaprendizagem das tarefas funcionais e, assim, pode-se maximizar a recuperação da independência funcional. Desta forma, essa afirmação pode ser verificada neste estudo, uma vez que os indivíduos que foram submetidos ao atendimento fisioterapêutico apresentaram um declínio na escala da funcionalidade menor que aquele observado no grupo controle. Este achado também corrobora com a afirmação de Oliveira (2013) de que a fisioterapia é eficaz no processo de reabilitação de indivíduos que apresentam patologias neurológicas, uma vez que os objetivos serão minimizar a disfunção e estimular a funcionalidade; consequentemente, irá melhorar a qualidade de vida.

O tratamento dos pacientes acometidos pela ELA ainda é um pouco desafiador. Dessa forma, tem sido escolhida uma abordagem multidisciplinar para que os indivíduos apresentem melhores prognósticos (TRAYNOR et al., 2003). Várias opçóes de tratamento vêm aparecendo ao longo dos anos para melhorar a qualidade de vida e a sobrevivência de indivíduos acometidos pela ELA; dentre essas abordagens, está a fisioterapia (LINDEN JUNIOR, 2013).

Em meio aos recursos que a fisioterapia pode utilizar como opção para o processo de reabilitação, podemos destacar os exercícios que previnam deformidades, que estimulem a mobilidade e retardem a dependência funcional inerente a esta doença. Também é possível optar pelos exercícios resistidos, já que inatividade e o desuso podem aumentar a fraqueza muscular, sobrepondo-se à fraqueza causada pela própria doença. Outra proposta de tratamento é a mobilização articular, uma vez que o movimento é fundamental para o funcionamento articular e a falta do mesmo pode ocasionar dor e alteraçóes articulares. Nos pacientes com ELA, a mobilização articular passiva é de extrema importância para manter a mobilidade articular e, consequentemente, uma maior independência funcional (MAYADEV et al., 2008).

Assim, é possível entender que a fisioterapia é um grande coadjuvante no processo de reabilitação dos indivíduos acometidos pela ELA e isso explica o que foi observado no presente estudo, pois os indivíduos que foram submetidos ao atendimento fisioterapêutico mostraram, quando comparados ao grupo controle, melhores escores no que diz respeito à manutenção do nível funcional na escala de funcionalidade específica para ELA. Esse resultado ainda é mais bem compreendido a partir da afirmação de Linden Junior (2013), que sugere que a fisioterapia tem um papel importante no tratamento de pacientes com ELA e que os déficits que surgem ao longo da doença podem ser minimizados utilizando-se métodos e técnicas fisioterapêuticas.

\section{Conclusão}

Considerando-se a recomendação para abordagem multidisciplinar na ELA, a fisioterapia pode ser um grande coadjuvante no retardo de declínio funcional em pacientes com ELA. Ressalta-se também a importância de escalas específicas para ELA, considerando-se o caráter progressivo e heterogêneo da doença. Este estudo apresenta como limitação não ter sido realizado um acompanhamento sobre outros tratamentos de ordem multidisciplinar aos quais estes pacientes poderiam estar sendo submetidos e que poderiam interferir nos resultados encontrados neste estudo. Além disso, não foi considerado o tempo de diagnóstico e a evolução clínica, no que diz respeito à análise estatística.

Portanto, sugere-se que novos estudos com essa temática sejam desenvolvidos, a fim de respaldar cientificamente os benefícios que a fisioterapia pode causar a esses pacientes.

\section{Referências}

ANEQUINI, I. P. et al. Avaliação das atividades da ABRELA: orientações oferecidas, expectativas atingidas? Revista Neurociências, São Paulo, v. 14, n. 2, p. 191-197, 2006.

BANDEIRA, F. M. et al. Quality of life evaluation in Amyotrophic Lateral Sclerosis (ALS) patients in Brasilia. Revista Neurociências, São Paulo, v. 18, n. 2, p. 133-138, 2010.

BROOKS, B. R. et al. El Escorial revisited: revised criteria for the diagnosis of amyotrophic lateral sclerosis. Amyotrophic Lateral Sclerosis and Other Motor Neuron Disorders, Londres, v. 1, n. 5, p. 293-299, 2000. PMID: 11464847. 
CEDARBAUM, J. M. et al. The ALSFRS-R: a revised ALS functional rating scale that incorporates assessments of respiratory function. Journal of the Neurological Sciences, Amsterdá, v. 169, n. 1-2, p. 13-21, 1999.

CEDARBAuM, J. M.; STAMBLER, N. Performance of the Amyotrophic Lateral Sclerosis Functional Rating Scale (ALSFRS) in multicenter clinical trials. Journal of the Neurological Sciences, Amsterdã, v. 152, n. 1, p. 1-9, 1997.

CESÁRIO, C. M. M.; PENASSO, P.; OLIVEIRA, A. P. R. Impacto da disfunção motora na qualidade de vida em pacientes com acidente vascular encefálico. Revista de Neurociências, São Paulo, v. 14, n. 1, p. 6-9, 2006.

COMIM, C. M. et al. Analysis of central fatigue and its impact in the quality of life of patients with neuromuscular diseases. Fisioterapia Brasil, Rio de Janeiro, v. 10, n. 5, p. 308-313, 2009.

COSMO, S. A.; LUCENA, R. C. S.; SENA, E. P. Aspectos clínicos determinantes da capacidade funcional na Esclerose Lateral Amiotrófica. Revista de Ciências Médicas e Biológicas, Salvador, v. 11, n. 2, p. 134-139, 2012.

JENKINSON, C. et al. Development and validation of short measure of health status for individuals with Amyotrophic Lateral Sclerosis/motor neuron disease: the ALSAQ-40. Journal of Neurology, Berlim, v. 246, n. 3, p. 16-21, 1999.

KAUFMANN, P. et al. The ALSFRS predicts survival time in an ALS clinic population. Neurology, Nova Iorque, v. 64, n. 1, p. 38-43, 2005.

KEITH, R. A. et al. The functional Independence measure: a new tool for rehabilitation. Advances in Clinical Rehabilitation, Nova Iorque, v. 1, p. 6-18, 1987.

KIMURA, F. et al. Progression rate of ALSFRS-R at time of diagnosis predicts survival time in ALS. Neurology, Nova Iorque, v. 66, n. 2, p. 265-267, 2006.

KRUPP, L. B. et al. The fatigue severity scale: application to patients with multiple sclerosis and systemic lupus erythematosus. Archives of Neurology, Chicago, v. 46, n. 10, p. 1121-1123, 1989.

LINDEN JUNIOR, E. Abordagem Fisioterapêutica na Esclerose Lateral Amiotrófica: artigo de atualização. Revista Neurociências, São Paulo, v. 21, n. 2, p. 313-318, 2013. http://dx.doi.org/10.4181/RNC.2013.21.803.6p

MAYADEV, A. S. et al. The amyotrophic lateral sclerosis center: a model of multidisciplinary management. Physical Medicine and Rehabilitation Clinics of North America, Philadelphia, v. 19, n. 3, p. 619-631, 2008.

MIANO, B. et al. Inter-evaluator reliability of the ALS functional rating scale. Amyotrophic Lateral Sclerosis and
Other Motor Neuron Disorders, Londres, v. 5, n. 4, p. 235 239, 2004.

MILLER, R. G. et al. Consensus guideline for the design and implementation of clinical trials in ALS. Journal of the Neurological Sciences, Amsterdã, v. 169, n. 1-2, p. 2-12, 1999.

MITCHEK, J. D.; BORASIO, G. D. Amyotrophic lateral sclerosis. The Lancet, London, v. 369, n. 9578, p. 2031-2041, 2007.

OHASHI, Y. et al. Study of functional rating scale for amyotrophic lateral sclerosis: revised ALSFRS (ALSFRS-R) Japanese version. No To Shinkei, Tóquio, v. 53, n. 4, p. 346-355, 2001.

OLIVEIRA, D. S. Análise do perfil epidemiológico de pacientes com acidente vascular encefálico atendidos na clinica escola de saúde do Unifor-MG. 2013. 61 f. Monografia (Graduaçáo em Fisioterapia) - Centro Universitário de Formiga, Minas Gerais, 2013

PEREIRA, M. G.; DUARTE, S. Fadiga intensa em doentes com lúpus eritematoso sistémico: estudo das características psicométricas da escala da intensidade da fadiga. Psicologia, Saúde \& Doenças, Lisboa, v. 11, n. 1, p. 121136, 2010.

POZZA, A. M. et al. Physiotherapeutic conduct in amyotrophic lateral sclerosis. São Paulo Medical Journal, São Paulo, v. 124, n. 6, p. 350-354, 2006.

SHUMWAY-COOK, A.; WOOLLACOTT, M. H. Controle motor: teoria e aplicaçóes práticas. Barueri: Manole, 2003.

STAMBLER, N. The Amyotrophic Lateral Sclerosis Functional Rating Scale. Assessment of activities of daily living in patients with amyotrophic lateral sclerosis. The ALS CNTF treatment study (ACTS) phase I -II Study Group. Archives of Neurology, Chicago, v. 53, n. 2, p. 141147, 1996.

TRAYNOR, B. J. et al. Effect of a multidisciplinary amyotrophic lateral sclerosis (ALS) clinic on ALS survival: a population based study, 1996-2000. Journal of Neurology Neurosurgery and Psychiatry, London, v. 74, n. 9, p. 1258 1261, 2003. http://dx.doi.org/10.1136/jnnp.74.9.1258.

VAN DEN BERG, J. P. Multidisciplinary ALS care improves quality of life in patients with ALS. Neurology, Nova Iorque, v. 65, n. 8, p. 1264-1267, 2005.

WIJESEKERA, L. C.; LEIGH, P. N. Amyotrophic lateral sclerosis. Orphanet Journal of Rare Diseases, London, v. 4, n. 3, p. 1-22, 2009.

YORKSTON, K. M. et al. Manangement of speech end swallowing disorders in degeneratives diseases. Texas: Pro-ed, 2004.

\section{Contribuição dos Autores}

Gabriele Natane de Medeiros Cirne realizou a coleta dos dados, Luana Augusta Pimenta Bezerra redigiu o texto, Roberta de Oliveira Cacho realizou a redação do texto e análise estatística, Thaiana Barbosa Ferreira revisou, traduziu e submeteu o texto, Fabrícia Azevêdo da Costa Cavalcanti orientou, desenvolveu a metodologia e revisão. Todas as autoras aprovaram a versão final do texto. 